\title{
Experimental Studies of Forward and Backward Whirls of Drill-String
}

\author{
Marcin Kapitaniak $^{\mathrm{a}}$, Vahid Vaziri ${ }^{\mathrm{a}}$, Joseph Páez Chávez ${ }^{\mathrm{b}, \mathrm{c}}$, Marian Wiercigroch $^{\mathrm{a}, *}$ \\ ${ }^{a}$ Centre for Applied Dynamics Research, School of Engineering, University of Aberdeen, Aberdeen AB24 3UE, UK \\ ${ }^{b}$ Center for Applied Dynamical Systems and Computational Methods (CADSCOM), Faculty of Natural Sciences and \\ Mathematics, Escuela Superior Politécnica del Litoral, P.O. Box 09-01-5863, Guayaquil, Ecuador \\ ${ }^{c}$ Center for Dynamics, Department of Mathematics, TU Dresden, D-01062 Dresden, Germany
}

\begin{abstract}
In this work we investigate forward and backward whirls of a drill-string using a novel experimental drilling rig [1] capable of reproducing major types of drill-string vibration, including stick-slip, bit-bounce and whirling. We focus our attention on whirling motion of the Bottom Hole Assembly (BHA) with a particular attention to the co-existence of forward and backward whirls. We present experimental results, showing for the first time co-existing whirling solutions and characterizing the parameter space in which different whirls can be observed. Those results are then used to calibrate a simple mathematical model, which can be used for further studies of whirling phenomena.

Keywords: Experimental studies, Drill-string dynamics; Lateral vibration; Forward and backward whirls; Co-existing attractors;
\end{abstract}

\section{Introduction}

During a downhole drilling excessive vibration can occur, which in most cases have a negative effect on the drilling process. Such vibration may lead to an accelerated wear and a premature damage of the expensive drilling equipment. Often different dynamic effects such as bit-bounce, stick-slip, forward and backward whirls may appear for the same drill-string arrangement. In this paper we focus on the whirling of the BHA inside the borehole, which is still not well understood and can lead to catastrophic failures of drill-strings. As depicted in Fig. 1, there are two types of whirling motion: forward and backward whirls, for which the direction of rotation of the BHA coincides (forward) or differs (backward) with the direction of whirling motion. From those two, the backward whirl is a bigger threat to the drilling process, as it induces high frequency vibration. Besides, lateral vibration can lead a drill-string to bend and as a consequence it can compromise the borehole stability $[2,3]$.

\footnotetext{
${ }^{*}$ Corresponding author.

Email addresses: m.kapitaniak@abdn.ac.uk (Marcin Kapitaniak), vahid.vaziri@abdn.ac.uk (Vahid Vaziri), jpaez@espol.edu.ec (Joseph Páez Chávez), m.wiercigroch@abdn.ac.uk (Marian Wiercigroch)
} 
(a)

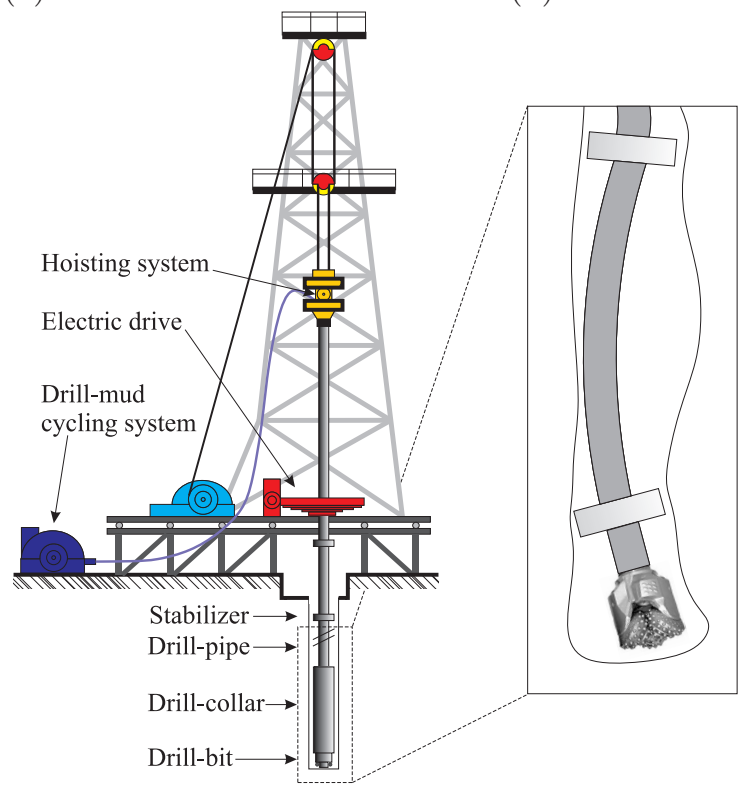

(c)

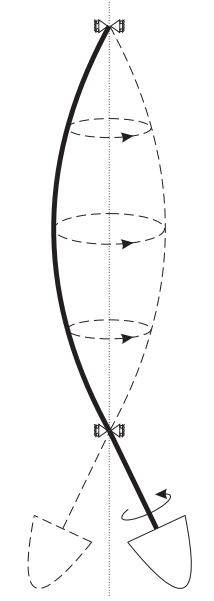

(d)

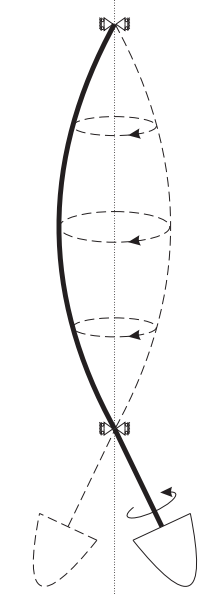

Figure 1: (a) A schematic showing a typical configuration of a drilling rig with most important components; (b) BHA section; (c) \& (d) schematics of BHA whirling motion, forward and backward respectively.

The whirling phenomena have attracted a considerable attention throughout the years including both numerical and experimental studies. Theoretically, most of the efforts have been on utilizing low dimensional models, based on a rotor concept, to mimic different types of whirling motion. A good summary of modelling efforts of different vibration modes is given in Ghasemloonia et al. [4]. In many cases this includes coupling of the lateral degrees-of-freedom (DOFs) with the torsional ones. Liao et al. [5] introduced four and five DOFs reduced models, coupling torsional and lateral dynamics, which proved to be in a good agreement with experimental results. The main solution of mitigating dangerous stick-slip oscillations is increasing the drill-string rotary speed, which often results in increased both lateral and axial vibration, whirling, impacts between a drill-string and a borehole, as well as parametric instabilities and bit-bounce [6].

One of the major reasons for whirling phenomenon is the imbalance (for example when the centre of gravity does not coincide with the centre of rotation), that results in its bowing around the borehole [5]. This results in an enlargement of the borehole and may lead to catastrophic failures in the system. In terms of modelling, the whirling has been analysed using unbalanced rotor systems [5, 7-9], which represents a simplification of the complex whirling phenomena encountered in downhole drilling problems. It has been broadly investigated in the past using different approaches, which include Jeffcot rotor models (e.g. $[7,10])$ as well as Finite Element models (e.g. [11, 12]). An interesting model has been introduced in [13], where authors introduce a digital filters based model of the drill-string, that takes into account 
nonlinear characteristics of drill-bit cutting interface and contact with the borehole, which is able to both stick-slip as well as forward/backward whirls. The asymmetry of the shaft is one of the main reasons for whirling phenomenon [14-16]. However, it has been reported in [17] that whirling can be also affected by the drill-bit rock interactions as well as friction between the BHA and the borehole [18].

There have been many attempts to investigate drill-string dynamics experimentally on various rigs having different capabilities. Warren [19] described a large scale experimental rig, capable of operating under conditions comparable to those observed in the field, whereas Hanson [20] reported a study of whirling tendencies of polycrystalline diamond compact (PDC) drill-bits. Another example of a large scale rig has been described by Halsey et al. [21], where a study of torsional vibration of the drill-string in a nearly vertical, 1000 meter deep borehole has been analysed. Due to the limited resources and space restrictions, the rigs developed in the academic institutions have been much smaller in size. In most of them, a drill-string is a slender steel structure, driven by an electric motor from the top, whereas the BHAs are represented as cylinders. Those rigs do not use real drill-bits but simulate the drill-bit rock interactions through different shakers and brakes. Examples of such drilling rigs can be found in [22], [23], [24] and [5], which is contrast to the work by Hoffmann [25], where actual rock samples were drilled. In [26] the authors describe a newly built scaled rig for analyzing drill-string vibration, which comprises of a rotating shaft between two stabilizers and is capable of replicating lateral vibration.

The main purpose of the present work is the experimental study of whirling phenomenon, in order to gain some further insight and to develop the calibrated mathematical models capable of accurately predicting the dynamical behaviour. The study is supported by a brief analysis with a simple model, that allows to expand the current understanding of the conditions, that trigger forward and (or) backward whirling motion. Ultimately, this work should contribute to development of simple and easily applicable methods to effectively control dynamics of the BHA dynamics.

The structure of this paper is as follows. In Section 2, we describe in details the experimental rig used to study whirling phenomenon, as well as specify conditions of the experiment. As a next step, Section 3 presents an experimental procedure and analysis of the system, providing examples of different possible types of whirling motion, including chaotic and periodic whirling of both forward and backward types. Moreover, we identify regions in the parameter space, where those types of whirling behaviour are present. Furthermore, examples of co-existing whirling responses are provided. In Section 4, we present a simple low-dimensional rotor model, that is capable of replicating experimental observations. We demonstrate a good qualitative agreement between the proposed model and the experimental results. The analysis involves study of co-existing periodic (forward/backward) and chaotic whirling responses 
and the influence of the system parameters determining their existence. Finally, conclusions are drawn in Section 5.

\section{Experimental Rig for Investigations of Drill-String Vibration}

\subsection{Aberdeen Drill-string Dynamics Experimental Rig}

In this section we describe briefly the experimental rig developed at the University of Aberdeen [1], that has been utilized to study different types of whirling motion. This includes the experimental setup presented in Fig. 2, which has been described in detail in [27, 28]. The most important feature of the experimental apparatus is its versatility, which means that depending on a chosen configuration, different types of drilling phenomena can be observed including stick-slip, bit-bounce and whirling. Moreover, the drilling rig is unique in using (i) flexible shafts to replicate torsional and bending properties of the drill-string, (ii) real commercial drill-bits (both PDC and roller-cone types) and real rock samples. The main components of the experimental setup, depicted in Fig. 2 can be grouped in three categories

- drill-string composed of flexible/rigid shaft, BHA, WOB disks, and the drill-bit,

- rock samples and cutting fluid circulation system and

- sensors, instrumentation and Data Acquisition System (DAQ).

As can be seen in Fig. 2, the drill-string is driven from the top using a modified pillar drilling machine, equipped with a 3kW electric motor that can provide up to $1032 \mathrm{rpm}$, depending on the chosen gearing configuration. In our experiments either a rigid or flexible shaft are driven from the top. The latter combines a high torsional rigidity with low bending stiffness to represent extreme slenderness of a drillstring. The internal structure of the flexible shaft comprises of several layers of thin wires, that are wound together. Typically, they are used to transmit power in rotating machines. At the other end, the flexible shaft is connected to the BHA section made of a heavy steel shaft, which is held in transversal direction using a loose bearing. The drilling machine is equipped with the spindle allowing axial movement of the top of the drill-string in the range 0 to $220 \mathrm{~mm}$, which is particularly useful when the helical bucking of a drill-string is studied $[28,29]$. An axial static force or a WOB is realized by placing steel disks on the top of the BHA providing WOB within the range 0.93 to $2.79 \mathrm{kN}$.

At the end of the BHA, commercial drill-bits are attached and placed on the top of rock sample, as depicted in detail in Fig. 3 (a). In our studies we have used various rocks including sandstone, granite, limestone. The versatility of the drilling rig allows to use different sizes of the drill-bits and rock samples. In Fig. 3 (b) examples of drilled rock samples (a cube of approximately $15 \times 15 \times 15 \mathrm{~cm}$ ) of granite and sandstone are presented. As can be seen in Fig. 2, the sample is placed inside the container, which has 

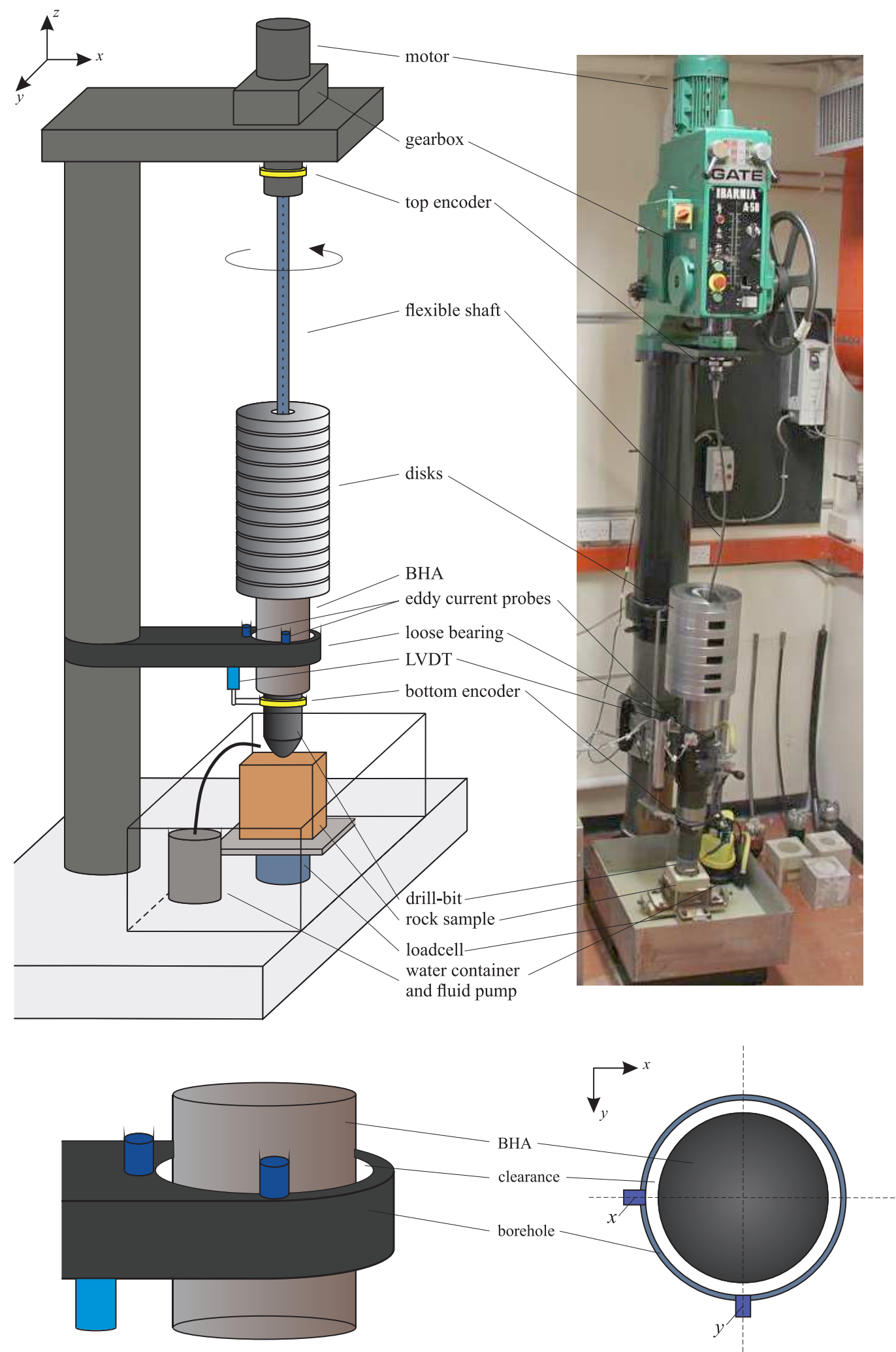

Figure 2: A schematic (left) and photograph (right) of the rig showing its main components such as BHA, flexible shaft, WOB disks, drill-bit, rock sample and motor. The instrumentation include a 4D load-cell, LVDT, top and bottom encoders and eddy current probes. Two lower panels show zoom-ups of the BHA and the borehole. 
(a)

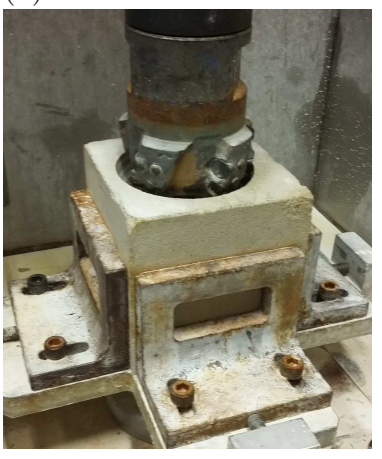

(b)

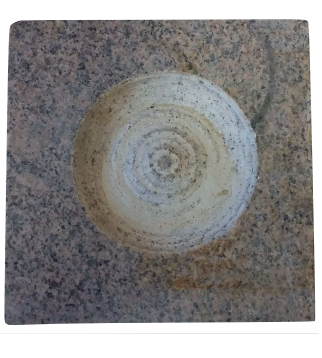

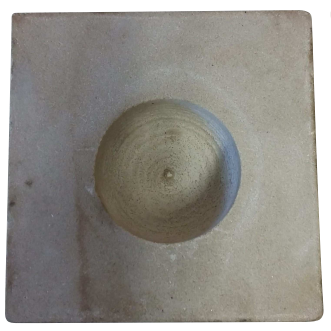

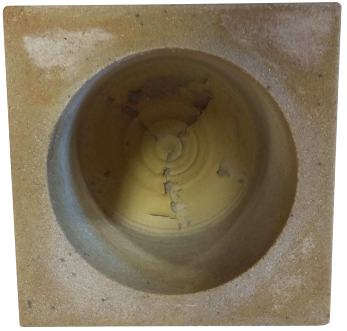

Figure 3: (a) A close up view on the drill-bit rock interface, (b) Examples of drilled granite and sandstone samples using various PDC drill-bits.

two levels to separate most of the cuttings from the water. We use a water based solution with anticorrosive agent, that is pumped directly into the drill-bit rock interface. After debris are removed from the borehole, the cuttings and the drilling fluid are driven by gravity to the lower level of the tank.

A variety of different sensors are used in the experimental setup, which allows us to conduct detailed measurements of the most important parameters of the drilling process. These include two rotary encoders to measure top and bottom speed, two eddy current probes to measure position of the BHA inside the borehole and a Linear Variable Differential Transducer (LVDT) to observe Rate of Penetration (ROP). The most advanced sensor in our setup is the four component dynamometer (Kistler 9272), placed directly below the rock sample, which allows to measure the Weight On Bit (WOB), Torque On Bit (TOB), and two forces acting in transversal directions $x$ and $y$ as depicted in Fig. 2. The ranges of measurement for TOB and WOB are: 0-200 Nm and 0-20 kN respectively.

\section{Experiments}

\subsection{Experimental procedure}

In order to observe whirling phenomenon, the configuration of the rig needs to be appropriately adjusted, which means that a radial clearance between the BHA and the loose bearing restricting motion of the BHA in transversal directions, has to be kept within a certain range (see bottom panels of Fig. 2). This is done by using a bush forming loose bearing to create an appropriate radial clearance of $1.25 \mathrm{~mm}$. The experimental procedure involves selection of an appropriate flexible shaft to replicate the dynamic behaviour in question. In the current work, majority of the experiments have been conducted using the flexible shaft of torsional stiffness $(28.07 \mathrm{Nm} / \mathrm{rad})$ and diameter of $15 \mathrm{~mm}$. As described in [27] a 3 7/8" PDC drill-bit and sandstone rock samples are used in this study, where the WOB is provided by means 
of steel discs and this parameter remains constant for one set of experiments. For a given set of system parameters (drill-bit, rock sample, flexible shaft and WOB), the experiments are run at different top speeds and the dynamic responses of the BHA are recorded. This is performed by using Labview based Data Acquisition System (DAQ), which allows to observe in real time readings from all the available sensors, as well as to save the data for subsequent data processing and analysis. In Fig. 4 we present an example family of time histories of a typical experiment for the configuration with 20 disks (each of mass $10.5 \mathrm{~kg}$ ). The drill-string is driven from the top with an angular velocity of $11 \mathrm{rad} / \mathrm{s}$, which is recorded and depicted in panel (c). Panels (a) and (b) depict the displacement of the BHA in transversal directions $x$ and $y$. As can be seen in panel (c), the top speed, as provided by the motor, has oscillatory characteristics of small amplitude oscillations, which has a direct effect on the response of the angular speed of the BHA, presented in panel (d). We observe here oscillations of peak to peak amplitude of $6 \mathrm{rad} / \mathrm{s}$, which is a direct consequence of the low stiffness of the flexible shaft. Note, that the lateral and torsional oscillations have different frequencies, as can be seen when comparing the time histories of $x(t), y(t), \dot{\varphi}_{t}(t), \dot{\varphi}_{b}(t)$, shown in panels(a)-(d). The experimental rig is equipped with accuracy load-cell (Kistler 9272) capable of high quality force measurements including the axial force and resistive torque, presented in panels (e) and (f) respectively. As a result of varying speed of the BHA, we observe small amplitude variation in both $F_{z}$ and $T_{z}$, around their mean value. Additionally the load-cell used in the experimental setup gives us information about forces in transverse directions $F_{x}$ and $F_{y}$, shown in panels (g) and (h) respectively. 

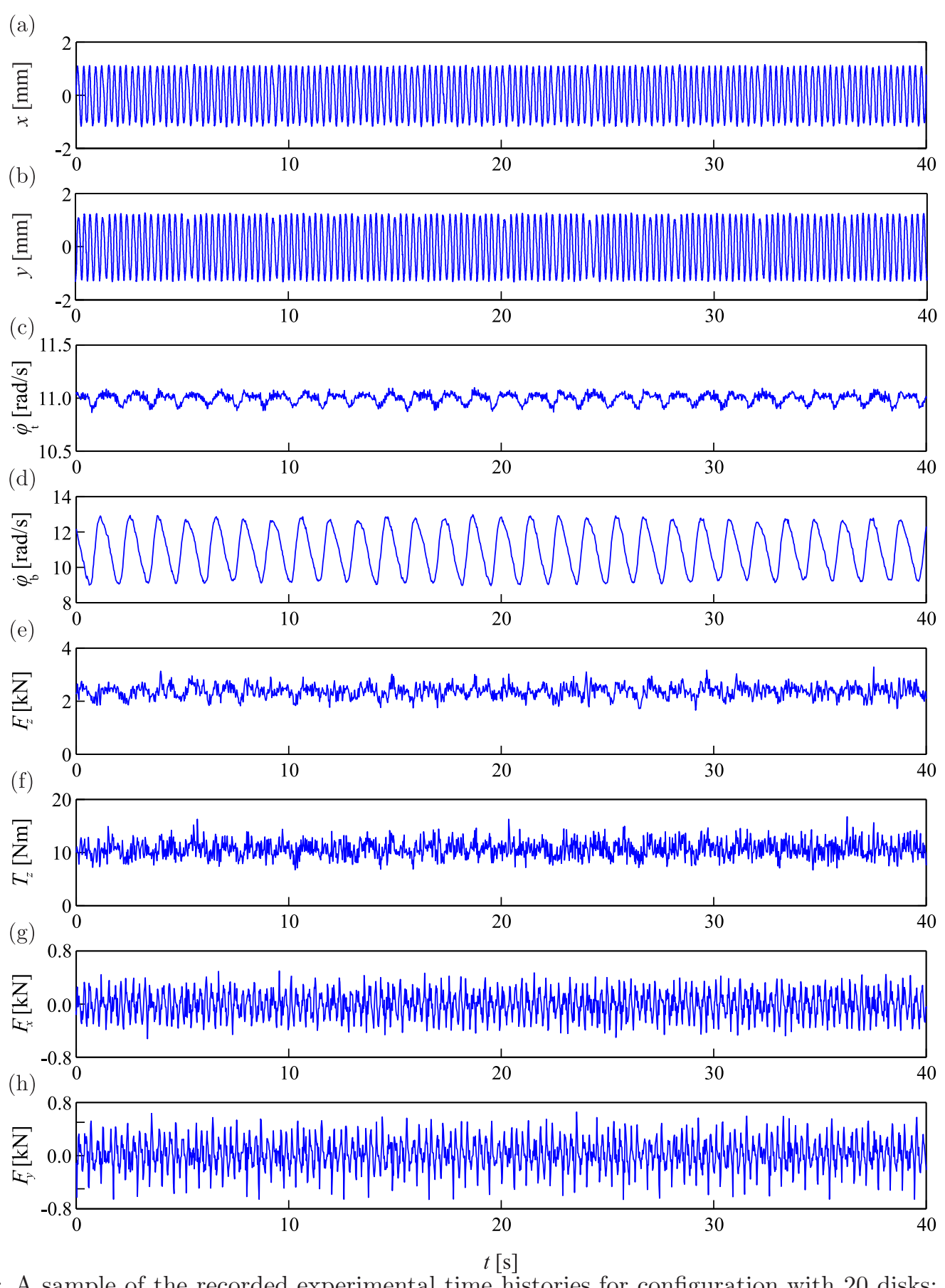

Figure 4: A sample of the recorded experimental time histories for configuration with 20 disks; (a) and (b) displacement of the BHA in the $x$ and $y$ direction respectively, (c) and (d) top and bottom speed, (e) $F_{z},(\mathrm{f}) T_{z}$, (g) and (h) transverse forces on $x$ and $y$ direction acting on the drill-bit. 


\subsection{Experimental study of different whirling motions}

In this section we conduct experimental studies of whirling phenomena, observing different responses including both forward and backward, as well as chaotic whirls. As described in Sec. 2, the motion of the BHA is measured using two eddy currents probes, that enable us to observe the planar trajectory of the BHA. An example of a typical measurements of this trajectory, for the configuration with 20 disks and angular speed of $7.88 \mathrm{rad} / \mathrm{s}$, is shown in Fig. 5. This response corresponds to a periodic backward whirl, which is identified using phase portraits $(\dot{x}, x),(\dot{y}, y)$ shown in right panels in Fig. 5.
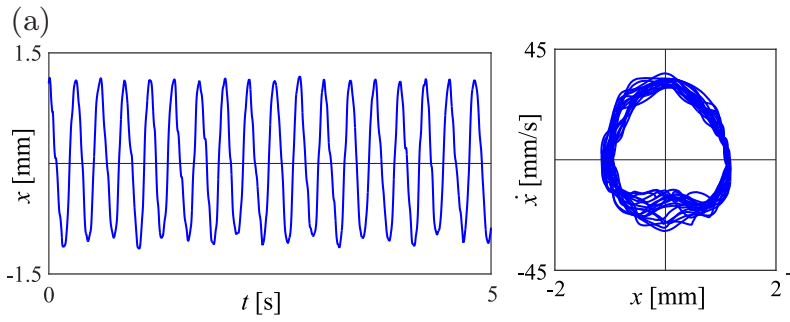

(b)

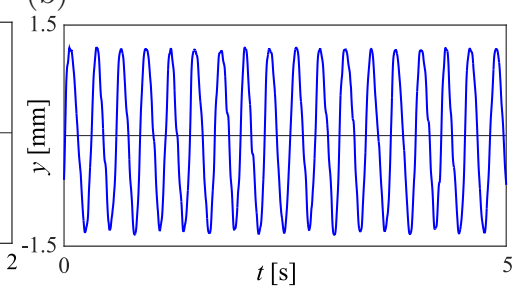

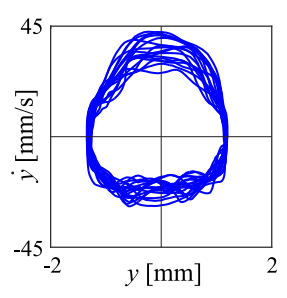

Figure 5: An example experimental time histories of lateral motion of the BHA in $x$ and $y$ directions, for the configuration with 20 disks and angular speed of $7.88 \mathrm{rad} / \mathrm{s}$ and corresponding phase portraits.

As the first step we investigate the effect of an angular velocity provided at the top by keeping fixed number of WOB disks and recording data for different values of angular speed of the BHA. Then the WOB is increased and the whole procedure is repeated. Fig. 6 depicts a two parameters diagram, showing different experimental whirling motion types. Three different types of whirling behaviour are recognized, based on the percentage of the trajectory points being outside the circle marked in black, as shown in Fig. 6 (b)-(d) (type $A<65 \%, 65 \%<$ type $B<85 \%$, type $C>85 \%$ ). Types $A$ and $B$ represent chaotic like whirling motions, whereas type $C$ represents periodic response, for which the BHA is in permanent contact with the borehole. The measurement points for different cases are denoted with dots in blue, cyan and red colors for types $A, B$ and $C$ respectively. The boundary regions are estimated based on the obtained measurement points. As can be seen in Fig. 6, the chaotic like whirling is dominant for lower values of angular velocity and WOB. As the WOB increases we observe more and more of periodic responses. The region of the periodic whirling expands as the WOB increases, so that even for lower angular velocities periodic whirling responses were found. Interestingly, inside the periodic whirling region, the forward whirling is dominant, although in some cases we were able to induce backward whirling, by changing the initial conditions or practically giving a kick to the BHA, what indicates a co-existence of these responses. This aspect of whirling motion will be discussed in details in the next section.

Interestingly enough, it is possible to obtain a response, for which we observe interchanging between a chaotic like and periodic whirling. Such a behaviour has been observed experimentally for the WOB 
(b)
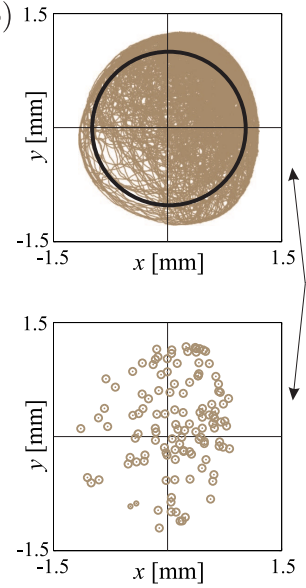

(a)
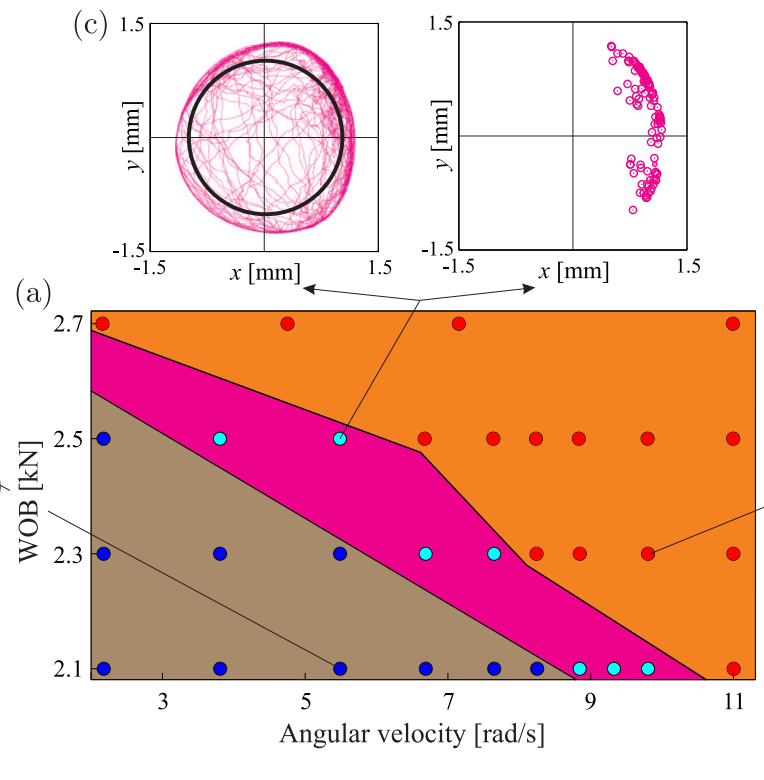

(d)
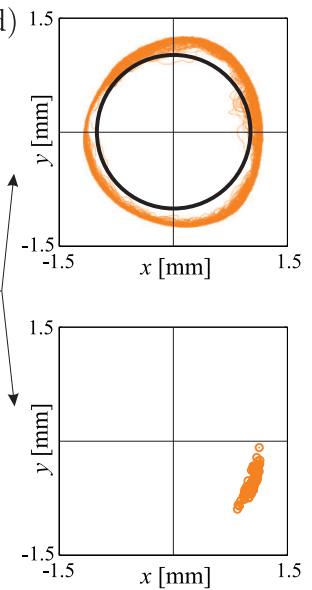

Figure 6: (a) Regions of different whirling behaviour as a function of applied WOB and angular velocity of the BHA. Trajectories of the BHA motion and corresponding Poincaré maps showing three types of whirling behaviour: (b) chaotic like motion (type $A$ ), (c) intermediate state (type $B$ ), (d) almost periodic motion (type $C$ ).

value of $1.95 \mathrm{kN}$ and is shown in Fig. 7 (a), which depicts a 3D representation of the trajectory of the BHA, in $x$ and $y$ directions, as a function of time. As can be seen, in the beginning we observe chaotic like whirling motion (marked in blue). In this case there are small oscillations of the angular velocity of the BHA, which is shown in Fig. 7 (b). Based on the planar displacement measurements of the BHA, an angular displacement of the BHA with respect to the borehole $\theta$ is computed. By comparing the slopes of $\theta$ and angular displacement at the top $\varphi_{t}$, it is evident that the first region shows a chaotic like forward whirl as the slopes have the same sign (See Fig. 7 (c)). Whirling direction seems to be sensitive to the varying angular velocity of the bit, as the BHA starts to move in a backward direction, as the $\dot{\varphi}_{t}$ increases. When the angular velocity of the BHA reaches the maximum value and starts to decrease, the whirling motion becomes chaotic like and the whirl direction switches from backward to forward. As can be seen in Fig. 7 (b), the motion of the BHA becomes periodic and backward again, as the angular velocity of the BHA increases. This clearly demonstrates a coupling between the torsional oscillations of the drill-string and the lateral vibration of the BHA, as has been widely reported in literature, e.g. [30].

\subsection{Co-existence of forward and backward whirling}

As described above, different types of whirling motion have been identified experimentally. Interestingly, inside the parameter space where periodic whirling dominates (orange region in Fig. 6), two 
(a)

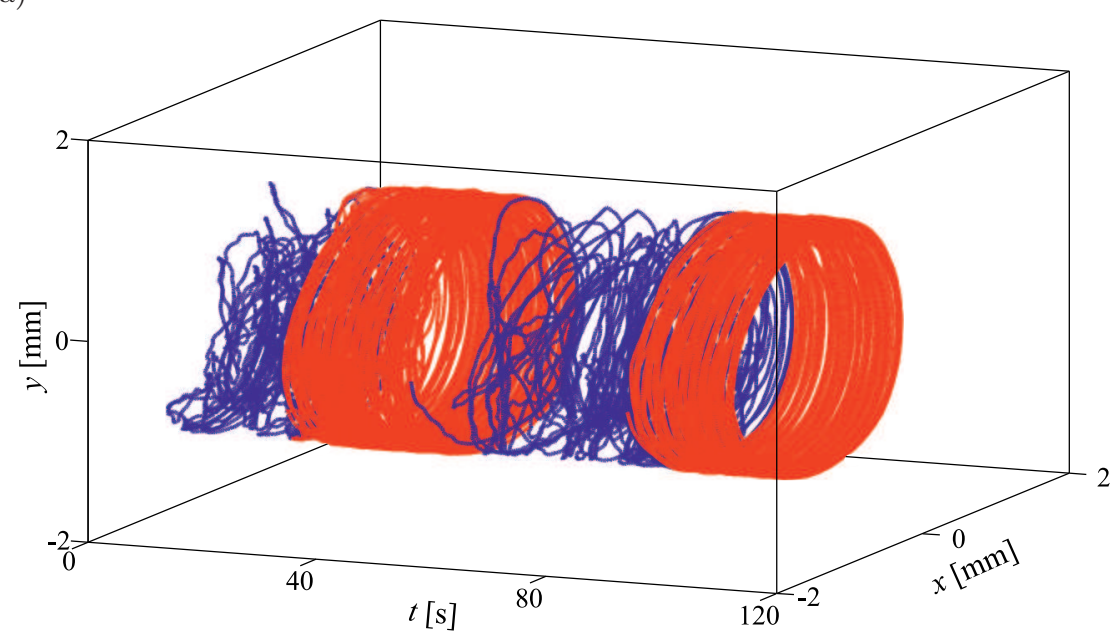

(b)

(c)
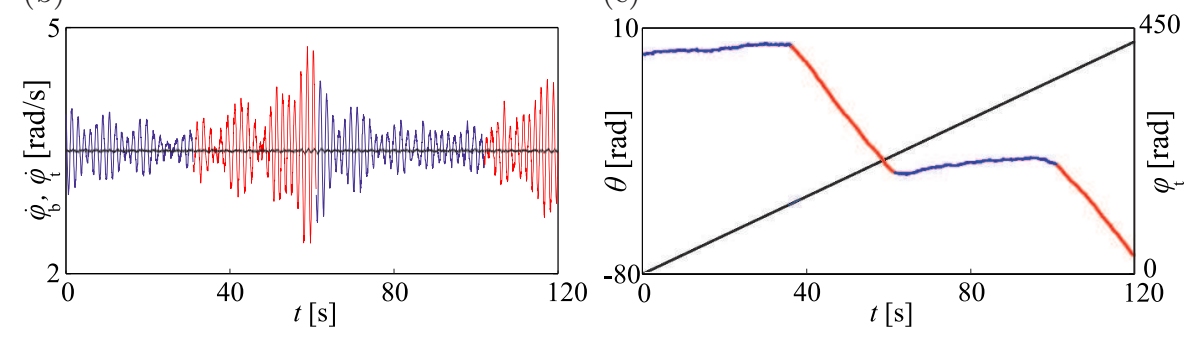

Figure 7: (a) Three dimensional representation of BHA trajectory with respect to time, showing interchanging regions of chaotic like forward and periodic backward whirls, (b) time history of angular velocity at the top, $\dot{\varphi}_{t}$ and the bottom, $\dot{\varphi}_{b}$, (c) time history of angular displacement at the top $\varphi_{t}$ (black) and whirling angular displacement of the BHA inside the borehole $\theta$, where forward and backward whirls are marked in blue and red respectively.

different types of periodic responses have been observed, forward and backward. They exist for the same set of parameters (WOB and angular speed at the top $\dot{\varphi}_{t}$ ), which clearly indicates co-existing responses.

As has been indicated earlier, in order to switch from one type of periodic response to another, one needs to perturb the initial conditions of the BHA (as described in Sec. 2.1). An example of such a response for $\mathrm{WOB}=2.59 \mathrm{kN}(22$ disks $)$ and angular velocity at the top $\dot{\varphi}_{t}=9.36 \mathrm{rad} / \mathrm{s}$ is shown in Fig. 8 . As has been explained in the previous section, the type of whirling motion is determined by comparing the angular displacement of the top $\varphi_{t}$ with the angular displacement of the BHA, $\theta$, which are depicted in panels (b) and (e) for backward and forward whirling motion, respectively. When comparing the orbits shown in panels (a) and (b), the qualitative responses are different, but both cases can be characterized as periodic whirling. In case of the backward whirling depicted in panel (a), one can conclude that the BHA makes one full turn inside the borehole, after which it loses contact with the wall, only to contact it again at the opposite side.

Interestingly there is a considerable difference in amplitude between the angular velocity of the BHA 
(a)

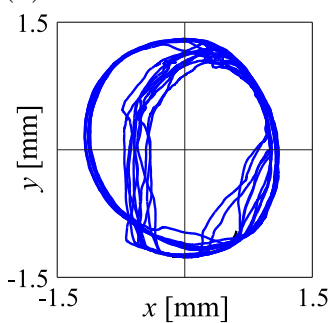

(d)

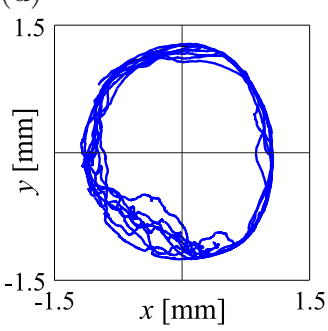

(b)

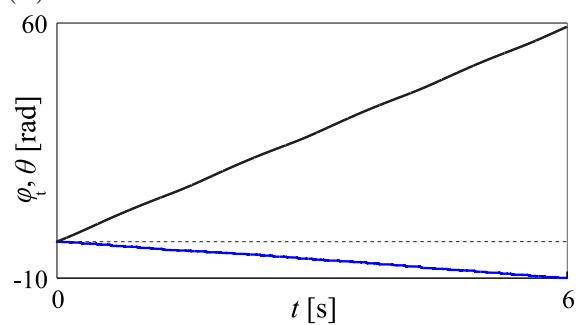

(e)

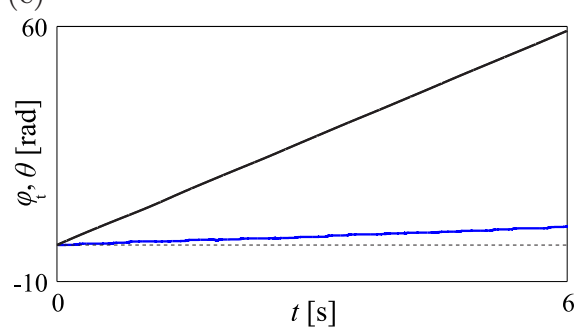

(c)

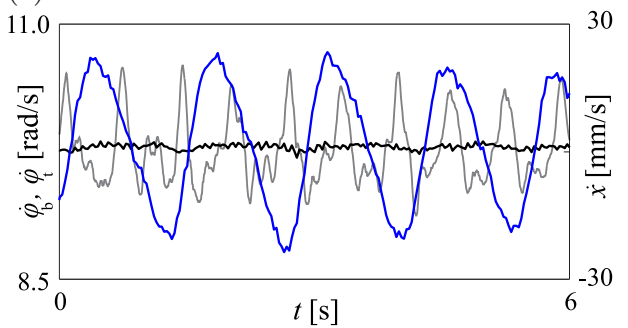

(f)

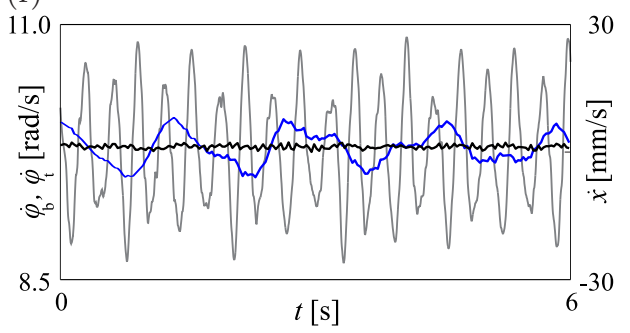

Figure 8: Comparisons between co-existing periodic backward and forward whirling as observed for $\mathrm{WOB}=2.59 \mathrm{kN}$, what corresponds to arrangement with 22 disks. Panels (a) and (b) show trajectories, where panels (c) and (d) compare angular displacement of top $\varphi_{t}$ vs. angular displacement of the BHA, $\theta$ and panels (e) and (f) show the time histories of angular velocity of top, $\dot{\varphi}_{t}$ (marked in black) and bottom, $\dot{\varphi}_{b}$ (marked in blue), as well as lateral velocity, $\dot{x}$ (marked in grey) for backward and forward whirl respectively.

$\dot{\varphi}_{b}$ for backward and periodic whirling, even though the top angular velocity, $\dot{\varphi}_{t}$, is constant (See black curve in panels (c) and (f)). This points out to the existence of coupling between lateral and torsional modes of vibration, as well as the fact that backward whirling is more dangerous than the forward one, due to the higher amplitude of torsional oscillations, which in turn results in higher stresses in the drill-string.

\section{Mathematical modelling}

In order to understand the mechanism of the co-existence of forward and backward whirls we introduce a simple mathematical model of the analyzed experimental setup. In essence, the model is similar to a Jeffcott rotor model with a snubber ring, which has been studied extensively in the literature, e.g. [7, 31].

The model depicted in Fig. 9 consists of a disc having mass $M$ and radius $a$, which spins inside the borehole with a constant angular velocity $\Omega$, around its centroid (point $\mathrm{O}_{2}$ ). The position of the disc inside the borehole is described using polar coordinates $r$ and $\theta$, that constitute a two degrees-of-freedom model. To model the excitation a small imbalance of the disc, $\rho$, is assumed. The disc has a viscous damping coefficient $c$ in radial direction and stiffness $k_{1}$. The radial clearance between the disc and the borehole is $\gamma$. Due to the fact that two modes of operation are present (contact and non-contact), there are two sets of equations, which need to be switched. We model the contact of the BHA with the borehole 


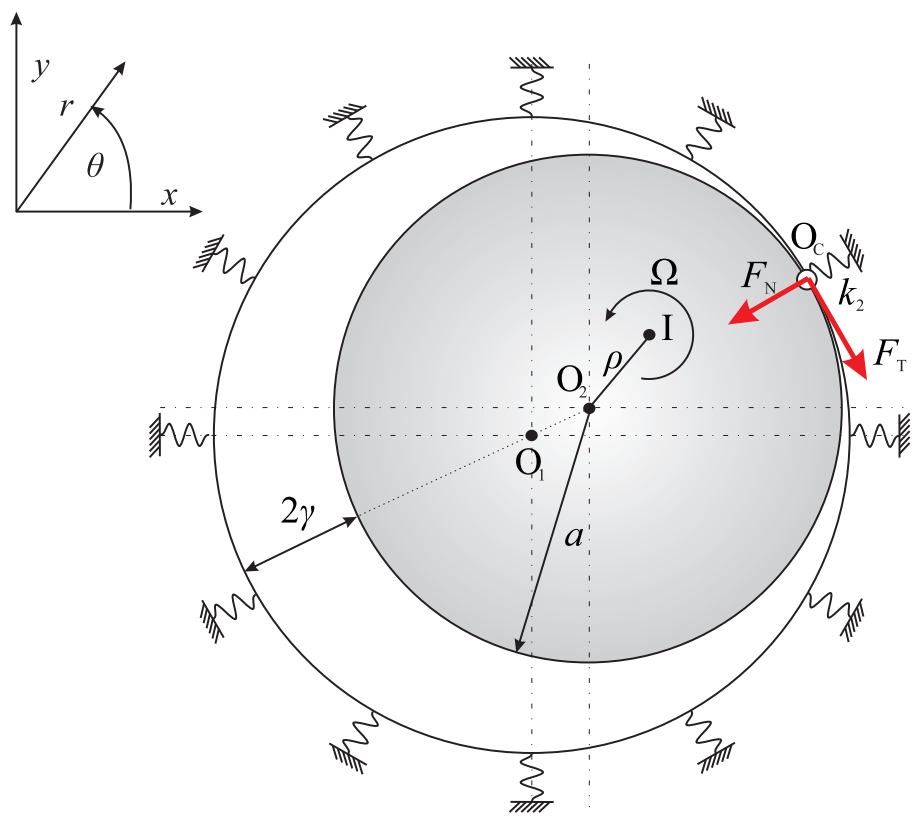

Figure 9: A schematic to model a planar motion of the BHA using the two degrees-of-freedom rotor system, represented as a disc of mass $M$ rotating around its centroid (point $\mathrm{O}_{2}$ ) with velocity $\Omega$ inside the borehole of radial clearance $\gamma$.

using a spring of high stiffness $k_{2}$ and dry friction $\mu$ between disc and the borehole in tangential direction, what means that at the contact point $\left(\right.$ Point $\mathrm{O}_{\mathrm{C}}$ ) between the disc and the borehole following forces:

$$
\begin{aligned}
& F_{\mathrm{N}}=k_{2}(r-\gamma) H(r-\gamma), \\
& F_{\mathrm{T}}=\mu k_{2}(r-\gamma) H(r-\gamma) \operatorname{sgn}(r \dot{\theta}+a \Omega),
\end{aligned}
$$

in normal and tangential directions act on the rotor disc. This leads to a following set of equations:

$$
\left\{\begin{array}{l}
M \ddot{r}-M r \dot{\theta}^{2}+k_{1} r+c \dot{r}=M \Omega^{2} \rho \cos (\Omega t-\theta)-k_{2}(r-\gamma) H(r-\gamma), \\
M r \ddot{\theta}+2 M \dot{r} \dot{\theta}+c r \dot{\theta}=M \Omega^{2} \rho \sin (\Omega t-\theta)-\mu k_{2}(r-\gamma) H(r-\gamma) \operatorname{sgn}\left(v_{c}\right) .
\end{array}\right.
$$

The switching conditions for the contact velocity $v_{c}=r \dot{\theta}+a \Omega$ and the contact are modelled by a Heaviside's step function. In other words this means that the disc can be in contact $(C)$ or noncontact $(N)$ state, depending on the value $r-\gamma$. Different set of equations is required when the contact velocity $v_{c}=0$, what means that the disc sticks to the wall. In such a case the equations of motion will take the following form: 


$$
\left\{\begin{array}{l}
M \ddot{r}-M r \dot{\theta}^{2}+k_{1} r+c \dot{r}=M \Omega^{2} \rho \cos (\Omega t-\theta)-k_{2}(r-\gamma) H(r-\gamma), \\
\dot{\theta}=\frac{-a \Omega}{r} .
\end{array}\right.
$$

\subsection{Numerical analysis}

As described in Sec. 3.2, we consider only four different configurations of WOB: $2.06 \mathrm{kN}, 2.28 \mathrm{kN}$, $2.49 \mathrm{kN}$ and $2.71 \mathrm{kN}$, which correspond to an arrangement with 16, 18, 20 and 22 disks, respectively. The stiffness $k_{1}$ of the radial bearing is obtained through analysing free vibration tests, performed for the considered cases of WOB configurations. From Table 1 we see that the equivalent stiffness and damping of the rotor is changing linearly with the mass of the rotor $M$. Other dimensional parameters of the rotor model are as follows: $k_{2}=5.3025 \times 10^{9} \mathrm{~N} / \mathrm{m}, \gamma=0.00125 \mathrm{~m}, \rho=0.0094 \mathrm{~m}, a=0.05 \mathrm{~m}, \mu=0.05$.

Table 1: Parameters of the rotor model corresponding to the experimental setup for different number of discs (WOB).

\begin{tabular}{|c|c|c|c|}
\hline Number of disks & $M[\mathrm{~kg}]$ & $k_{1}[\mathrm{kN} / \mathrm{m}]$ & $c[\mathrm{Ns} / \mathrm{m}]$ \\
\hline \hline 16 & 209.98 & 25.88 & 47.03 \\
\hline 17 & 221.20 & 27.04 & 48.51 \\
\hline 18 & 232.42 & 28.20 & 50.01 \\
\hline 19 & 243.12 & 29.01 & 51.16 \\
\hline 20 & 253.82 & 29.82 & 52.31 \\
\hline 21 & 265.04 & 30.47 & 53.52 \\
\hline 22 & 276.25 & 31.13 & 54.74 \\
\hline
\end{tabular}

The aim of this analysis is to show that the presented rotor model (Eq. 3) can be successfully applied to mimic the whirling phenomenon as observed experimentally (see Fig. 6), with a particular attention to co-existence of different types of whirling motion. Let's consider one example for the parameters $M=253.80 \mathrm{~kg}$ and $\Omega=8.5 \mathrm{rad} / \mathrm{s}$, which corresponds to the experimental conditions as used in Fig. 6 . We construct a map showing the dynamic responses of the system based on the initial conditions $r_{0}$ and $\theta_{0}$. This involves integrating Eqs 3 and 4 for different initial conditions from the following ranges, $r_{0} \in(0,1)$ and $\theta_{0} \in(0,2 \pi)$. The basin of attraction shown in Fig. 10 clearly demonstrates various whirling orbits, similar to those observed experimentally. Specifically, we have obtained the co-existence of forward (marked in red) and backward (marked in blue) whirls which can be also chaotic like (marked in grey). It appears that the periodic backward motion is dominant, but it co-exists with the forward chaotic whirl as well as much less probable forward periodic whirl. Co-existence of all those types of whirling behaviour is not a rare phenomenon and can be observed for other sets of parameters. Examples of three possible types of whirling responses are shown in Fig. 10. This short analysis allows us to conclude that only 
using a two-dimensional calibrated rotor model, we are able to replicate possible whirl types, as observed in the experimental studies.
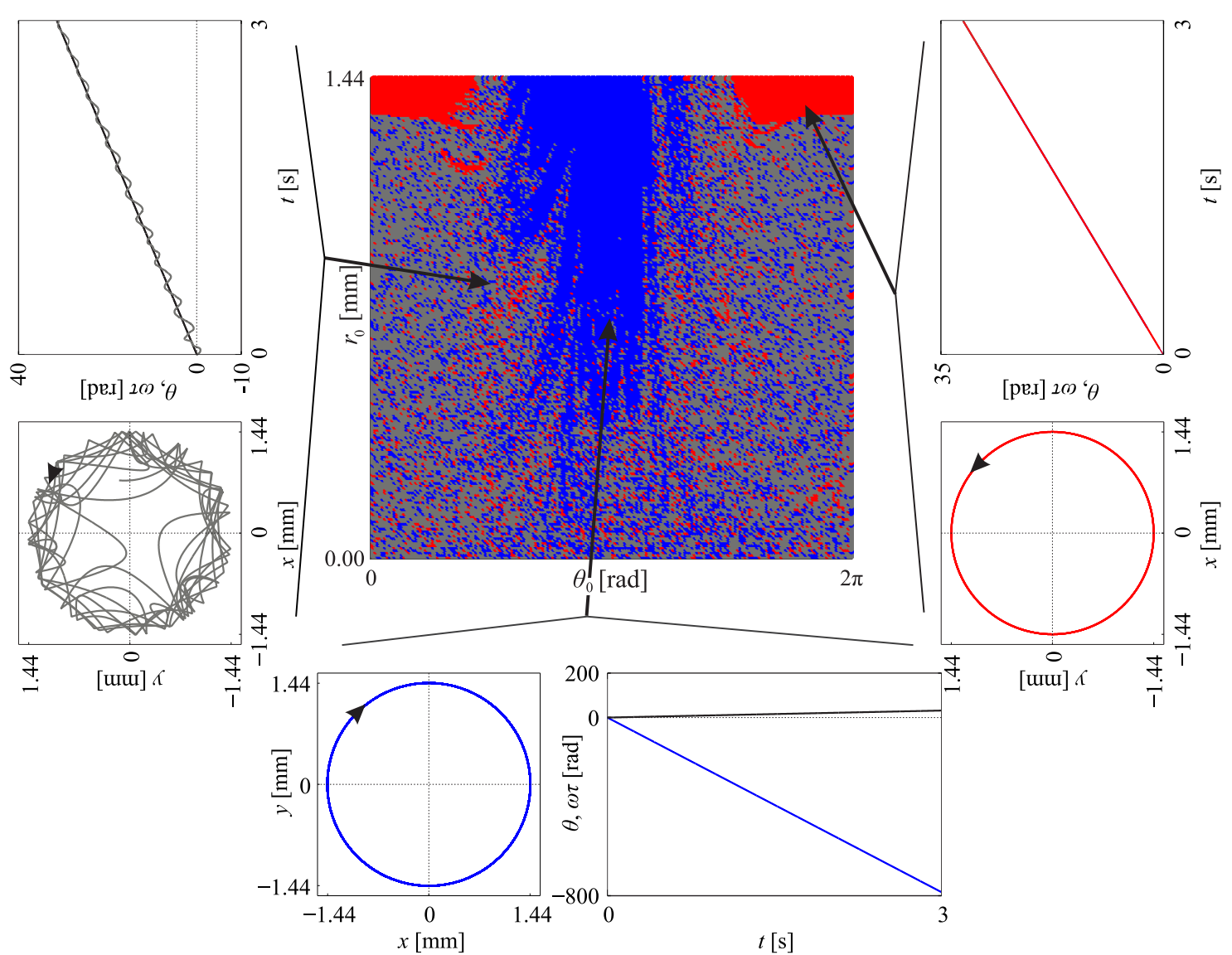

Figure 10: Map showing different types of possible co-existing whirling solutions depending on the initial condition of the rotor $r_{0}, \theta_{0}$ inside the borehole for parameters: $\Omega=8.50 \mathrm{rad} / \mathrm{s}, M=253.82 \mathrm{~kg}$, $\rho=10.80 \mathrm{~mm}, k_{1}=29.82 \mathrm{kN} / \mathrm{m}, k_{2}=5.30 \mathrm{GN} / \mathrm{m}, \mu=0.05$ and $a=57.76 \mathrm{~mm}$, where red, blue and grey colours represent periodic forward, periodic backward and chaotic forward whirl respectively. Examples of trajectory $(y, x)$ and time history of $\theta, \omega t$ are given for initial conditions: $r(0)=0.828 \mathrm{~mm}$, $\theta(0)=1.571 \mathrm{rad}$ (left panels), $r(0)=0.720 \mathrm{~mm}, \theta(0)=3.142 \mathrm{rad}$ (bottom panels) and $r(0)=1.368 \mathrm{~mm}$, $\theta(0)=5.655 \mathrm{rad}$ (right panels). 


\section{Conclusions and Future Work}

In this paper, forward and backward whirls on a versatile experimental drilling rig developed at the University of Aberdeen were studied. Firstly, we introduced the experimental stand, describing its possible configurations, sensors and instrumentation as well as the experimental procedure. One of the most important features of this experimental rig is that real commercial drill-bits and rock samples are used, contrary to most academic drilling rigs, which simulate drill-bit rock interactions using brakes and shakers.

Secondly, an experimental study to unveil the onset to various complex whirling responses was conducted. Those include periodic, chaotic, forward and backward whirls. Particular attention was given to study co-existence between different whirling responses including periodic and chaotic whirls, which has been observed for the first time experimentally. It has been demonstrated that by simply perturbing the initial condition one can switch between coexisting whirls, which was not a rare phenomena for the analyzed set of system parameters. Additionally, a parameter space diagram has been constructed experimentally to determine the effects of varying angular speed and mass of the BHA on type of motion observed. As both of those variables increase, the region of periodic whirl expands and the chaotic like whirl shrinks.

Next, a simple two degrees-of-freedom model to describe whirling phenomenon has been introduced and then calibrated to reflect the planar motion of the BHA. The model is based on a Jeffcott rotor with a snubber ring to mimic the lateral contact between a BHA and a borehole. The calibrated model allows to obtain qualitative agreement with experiments, allowing detailed study of coexisting whirling responses and their sensitivity to initial conditions. The numerical analysis together with the experimental studies allows to deepen our understanding of the whirling phenomenon.

In conclusion, in this paper we have presented a detailed experimental analysis of the whirling phenomenon and shown for the first time experimentally the co-existence of forward and backward whirling. A good qualitative agreement between the results from the experimental studies and the simple two dimensional model have been obtained. A further numerical analysis, using this model described briefly in this paper can be found in [28], where a particular attention is paid to the conditions that trigger forward and backward whirls with a view to devise methods of controlling the BHA dynamic behaviour.

\section{Acknowledgements}

The authors wish to thank BG Group plc for the financial support to this research. 


\section{References}

[1] M. Wiercigroch, "Modelling and Analysis of BHA and Drill-string Vibrations R\&D Project sponsored by the BG Group," 2010.

[2] A. Berlioz, J. D. Hogopian, R. Dufour, and E. Draoui, "Dynamic behavior of a drillstring: experimental investigation of lateral instabilities," Trans. ASME, J. Vib. Acoust, vol. 118, pp. 292-298, 1996.

[3] X. Zhu and W. Liu, "The effects of drill string impacts on wellbore stability," Journal of Petroleum Science and Engineering, vol. 109, pp. 217-229, 2013.

[4] A. Ghasemloonia, D. G. Rideout, and S. D. Butt, "A review of drillstring vibration modeling and suppression methods," Journal of Petroleum Science and Engineering, vol. 131, pp. 150 - 164, 2015.

[5] C. Liao, B. Balachandran, M. Karkoub, and Y. Abdel-Magid, "Drill-string dynamics: Reduced-order models and experimental studies," Journal of Vibration and Acoustics, vol. 133, no. (4), 2011.

[6] A. Yigit and A. P. Christoforou, "Stick-slip and bit-bounce interaction in oil-well drillstrings," Journal of Energy Resources Technology, vol. 128, 2006.

[7] J. Páez Chávez, V. Vaziri Hamaneh, and M. Wiercigroch, "Modelling and Experimental Verification of an Asymmetric Jeffcott Rotor with Radial Clearance," Journal of Sound and Vibration, vol. 334, pp. 86-97, 2015.

[8] J. Jansen, "Non-linear rotor dynamics as applied to oilwell drillstring vibrations," Journal of Sound and Vibration, vol. 147, no. 1, pp. 115-135, 1991.

[9] G. Van Der Heijden, "Bifurcation and chaos in drillstring dynamics," Chaos, Solitons and Fractals, vol. 3, no. 2, pp. 219-247, 1993.

[10] H. Wettergren and K.-O. Olsson, "Dynamic instability of a rotating asymmetric shaft with internal viscous damping supported in anisotropic bearings," Journal of Sound and Vibration, vol. 195, no. 1, pp. 75-84, 1996.

[11] S. Wang, Y. Wang, Y. Zi, and Z. He, "A 3D finite element-based model order reduction method for parametric resonance and whirling analysis of anisotropic rotor-bearing systems," Journal of Sound and Vibration, vol. 359, pp. 116 - 135, 2015. 
[12] A. Lazarus, B. Prabel, and D. Combescure, "A 3d finite element model for the vibration analysis of asymmetric rotating machines," Journal of Sound and Vibration, vol. 329, no. 18, pp. 3780 - 3797, 2010.

[13] T. Butlin and R. Langley, "An efficient model of drillstring dynamics," Journal of Sound and Vibration, vol. 356, pp. 100-123, 2015.

[14] A. Ghasemloonia, D. Rideout, and S. Butt, "Analysis of multi-mode nonlinear coupled axialtransverse drillstring vibration in vibration assisted rotary drilling," Journal of Petroleum Science and Engineering, vol. 116, pp. 36 - 49, 2014.

[15] S. M. Zamani, S. A. Hassanzadeh-Tabrizi, and H. Sharifi, "Failure analysis of drill pipe: A review," Engineering Failure Analysis, vol. 59, pp. 605 - 623, 2016.

[16] K. Ahmadi and Y. Altintas, "Stability of lateral, torsional and axial vibrations in drilling," International Journal of Machine Tools and Manufacture, vol. 68, pp. 63 - 74, 2013.

[17] Y. Kovalyshen, "A simple model of bit whirl for deep drilling applications," Journal of Sound and Vibration, vol. 332, no. 24, pp. 6321 - 6334, 2013.

[18] V. Gulyayev and L. Shevchuk, "Drill string bit whirl simulation with the use of frictional and nonholonomic models," Journal of Vibration and Acoustics, Transactions of the ASME, vol. 138, no. 1, 2016.

[19] T. M. Warren, "Drilling model for soft-formation bits," in SPE8438, 1979. Presented at the SPE 54th Annual Technical Conference and Exhibition, Las Vegas, September 23 -26.

[20] J. M. Hanson, "Dynamics modeling of PDC bits," in SPE/IADC29401, 1995. Presented at the SPE/IADC drilling conference and exhibition, Amsterdam, The Netherlands, February 26 - March 2 .

[21] G. W. Halsey, A. Kyllingstad, T. V. Aarrestad, and D. Lysne, "Drillstring torsional vibrations: Comparison between theory and experiment on a full-scale research drilling rig," in SPE15564, 1986. Presented at the SPE 61st Annual Technical Conference and Exhibition, New Orleans, Louisiana, October $5-8$.

[22] P. A. Patil and C. Teodoriu, "A comparative review of modelling and controlling torsional vibrations and experimentation using laboratory setups," Journal of Petroleum Science and Engineering, vol. 112 , pp. $227-238,2013$. 
[23] Y. A. Khuleif and F. A. Al-Sulaiman, "Laboratory investigation of drill-string vibration," in Proceedings of the IMech E Part C: Journal of Mechanical Engineering Science, vol. 223, pp. 2249-2262, 2009.

[24] N. Mihajlović, A. A. van Veggel, N. van de Wouw, and N. H., "Analysis of friction-induced limit cycling in an experimental drill-string system," ASME Journal of Dynamic Systems, Measurement, and Control, vol. 124, pp. 709-720, 2004.

[25] O. J. M. Hoffmann, Drilling induced vibration apparatus. PhD thesis, University of Minnesota, 2006.

[26] H. Westermann, I. Gorelik, J. Rudat, C. Moritz, M. Neubauer, J. Wallaschek, and O. Höhn, "A new test rig for experimental studies of drillstring vibrations," SPE Drilling and Completion, vol. 30, no. 2, pp. 119-128, 2015.

[27] M. Kapitaniak, V. Vaziri Hamaneh, J. Páez Chávez, and M. Wiercigroch, "Unveiling Complexity of Drill-String Vibrations: Experiments and Modelling," International Journal of Mechanical Sciences, vol. 101-102, pp. 324-337, 2015.

[28] M. Kapitaniak, Nonlinear Dynamics of Drill-Strings. PhD thesis, University of Aberdeen, 2015.

[29] M. Kapitaniak, V. Hamaneh, and M. Wiercigroch, "Torsional vibrations of helically buckled drillstrings: Experiments and FE modelling," Journal of Physics: Conference Series, vol. 721, no. 1, p. $012012,2016$.

[30] A. Chevallier, N. Politis, M. Payne, and P. Spanos, "Oil and gas well drilling: A vibrations perspective," Shock and Vibration Digest, vol. 35, no. 2, pp. 85-103, 2003.

[31] E. V. Karpenko, M. Wiercigroch, E. E. Pavlovskaia, and R. D. Neilson, "Experimental verification of Jeffcott rotor model with preloaded snubber ring," Journal of Sound and Vibration, vol. 298, pp. 907-917, 2006. 